\title{
ANÁLISE DOS PARÂMETROS OPERACIONAIS E DE RESERVATÓRIO APLICADO A RESERVATÓRIO DE GÁS COM BAIXA PERMEABILIDADE
}

\author{
F. de P. BESSA JUNIOR ${ }^{1}$, D. M. S de B. LIMA ${ }^{1}$, E. A. ARAÚJO ${ }^{1}$, A. A. R. DINIZ ${ }^{1}$, M. A. F. \\ RODRIGUES $^{1}$, W. MATA ${ }^{1}$ \\ ${ }^{1}$ Universidade Federal do Rio Grande do Norte, Departamento de Engenharia do Petróleo \\ E-mail para contato:jr_paiva20@hotmail.com
}

\begin{abstract}
RESUMO - Com o crescimento do consumo energético em todo o mundo, os reservatórios convencionais, chamados de reservatórios de "fácil exploração e produção" não estão atendendo a demanda energética mundial, fazendo-se necessário a exploração de reservas não convencionais. Como exemplo dessas reservas, temos os reservatórios do tipo Tight Gas, onde referem-se aos campos de arenito com baixa porosidade, na faixa de $8 \%$, e permeabilidade na faixa entre $0,1 \mathrm{mD}$ e $0,0001 \mathrm{mD}$, que acumulam consideráveis reservas de gás natural, podendo apresentar viabilidade econômica para explotação. O objetivo desse trabalho é analisar um planejamento fatorial completo de três variáveis com três níveis em função do percentual de gás recuperado em 40 anos. Os resultados mostraram que todos os parâmetros analisados individualmente no processo foram estatisticamente significativos, onde o número de fraturas foi o parâmetro que mais contribuiu para o aumento do fator de recuperação.
\end{abstract}

\section{INTRODUÇÃO}

Com o declínio dos reservatórios mundiais e com a dificuldade de encontrar novos campos em reservas convencionais, e com o aumento da demanda energética em todo o mundo, se faz necessário a busca pelas chamadas reservas não-convencionais, caracterizadas por suas baixas permeabilidade e porosidades. Estas reservas necessitam de maiores tecnologias empregadas para sua explotação, podendo ser extraídas a partir da aplicação da técnica de fraturamento hidráulico, técnica de estimulação que tem como objetivo injetar um fluido (fraturante) na formação, sob uma pressão suficientemente alta para causar a ruptura da rocha por tração.

Tight gas é o termo, que se refere ao gás natural contido em reservatórios de baixa permeabilidade requerendo o emprego de técnicas específicas para a sua produção comercial, tais como acidificação, fraturas em formações subterrâneas e, mais recentemente, utilização de poços horizontais e multilaterais.

A produção do tight gas é caracterizada por um curto período de alta produção com rápida queda, seguida por um longo período de baixa produção e declínio lento. Um poço de tight gas pode ter uma vida útil de até 50 anos, dependendo da capacidade de remoção de líquidos e do custo de produção com o avançar do tempo. A Figura 1 mostra uma microfotografia de um 


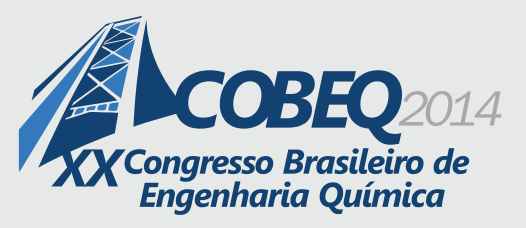

19 a 22 de outubro de 2014

Florianópolis/SC

arenito convencional e não convencional de um reservatório, no qual foi injetada tinta epóxi azul. As áreas azuis são os espaços porosos que contêm gás natural. O espaço poroso pode ser visto bem interligado (Figura do lado esquerdo) de forma que o gás seja capaz de fluir facilmente pela rocha, já a figura do lado direito mostra os seus poros distribuídos irregularmente onde a porosidade da rocha pode se vista bem menor do que o arenito convencional, dificultando assim a migração do fluido para outros espaços porosos.
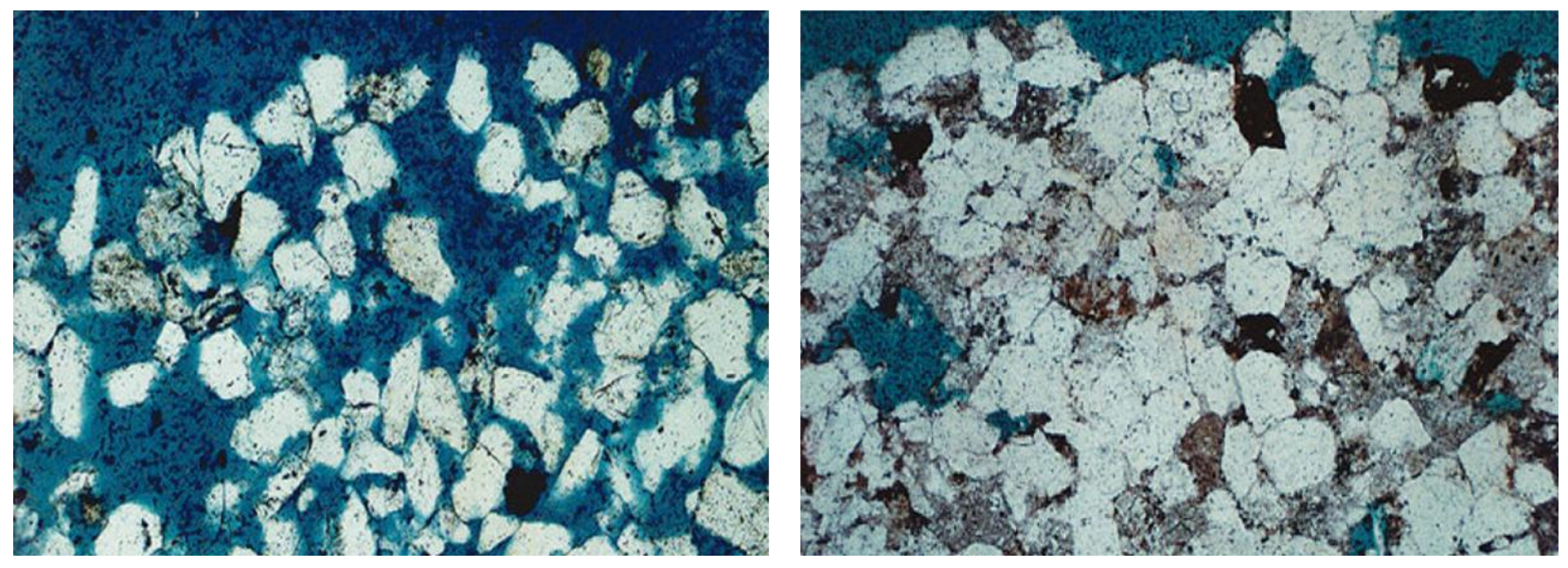

Figura 1 - Arenito Convencional e não-convencional de gás natural

(Fonte: Dutton et al, 1993).

O Brasil está prestes a dar os primeiros passos rumo ao desenvolvimento em grande escala da exploração de gás natural em terra. Segundo a Agência Nacional do Petróleo (ANP) existem grandes volumes de gás natural não-convencional em sete bacias sedimentares. Com base em estimativas preliminares da ANP, considerando um fator de recuperação médio de $70 \%$ dos reservatórios não-convencionais, o volume a ser explotado pode chegar a 10,1 trilhões de metros cúbicos de gás natural, número que só ficaria abaixo de Rússia, Irã, Qatar, Turcomenistão e Estados Unidos. O salto no ranking é gigantesco, o Brasil tinha a $32^{\mathrm{a}}$ maior reserva provada de gás no mundo em 2012, com 434 bilhões de metros cúbicos, segundo dados da Organização dos Países Exportadores de Petróleo (Opep). De forma a compreender o mecanismo da aplicação da técnica do fraturamento hidráulico em reservatório de gás com baixa permeabilidade foi proposto um estudo utilizando o simulador numérico IMEX 2012.10 da CMG (Computer Modelling Group). Neste trabalho foi analisada a influência de alguns parâmetros operacionais e de reservatório na recuperação de gás, usando um reservatório semi-sintético com características do Nordeste brasileiro. Neste estudo, foram realizadas análises de algumas variáveis operacionais tais como: quantidade e comprimento de fraturas, e o parâmetro de reservatório estudado foi a permeabilidade da matriz. Para avaliar a influência das variáveis independentes QF (Quantidade de fraturas), CF (Comprimento da fratura) e KZ (Permeabilidade da matriz) - sobre a variável dependente FR (fator de recuperação), elaborou-se um planejamento fatorial $3^{3}$, através do programa STATISTICA 6.0, totalizando 27 simulações. 


\section{Metodologia e Modelagem do Reservatório}

O modelo considerado foi um reservatório homogêneo semissintético. As dimensões da malha e as principais características do reservatório podem ser observadas na Tabela 1.

Tabela 1 - Dados do modelo de reservatório estudado

\begin{tabular}{cc}
\hline Propriedades & Valor \\
\hline Comprimento - i (m) & 250 \\
Largura - j (m) & 450 \\
Espessura - k (m) & 110 \\
Profundidade do topo do reservatório (m) & 4000 \\
Saturação de água conata (\%) & 20 \\
Saturação inicial de gás (\%) & 80 \\
Temperatura inicial $\left({ }^{\circ} \mathbf{C}\right)$ & 60 \\
Volume de gás original - $\left(\mathbf{m}^{3} \mathbf{s t d}\right)$ & $15,45 \times 10^{7}$ \\
\hline
\end{tabular}

Os parâmetros operacionais analisados neste trabalho foram a quantidade e o comprimento de fraturas e o parâmetro de reservatório foi a permeabilidade da matriz.. A Tabela 2 mostra os valores dos parâmetros analisados.

Tabela - 2 Parâmetros analisados

\begin{tabular}{cc}
\hline Parâmetros & Valores \\
\hline Quantidade de fratura (Unidade) & $1-5-9$ \\
Comprimento de fratura $(\mathbf{m})$ & $50-150-250$ \\
Permeabilidade da matriz $(\mathbf{m D})$ & $0,0005-0,001-0,002$ \\
\hline
\end{tabular}

Na Figura 2 (a esquerda), pode ser observada a modelagem da fratura dentro do reservatório, com as seguintes dimensões: $30 \mathrm{~m}$ de altura e $150 \mathrm{~m}$ de comprimento. $\mathrm{O}$ poço produtor é horizontal e possui um comprimento de $400 \mathrm{~m}$. Na mesma Figura (a direta) mostra as dimensões do reservatório, através da representação 3D. 

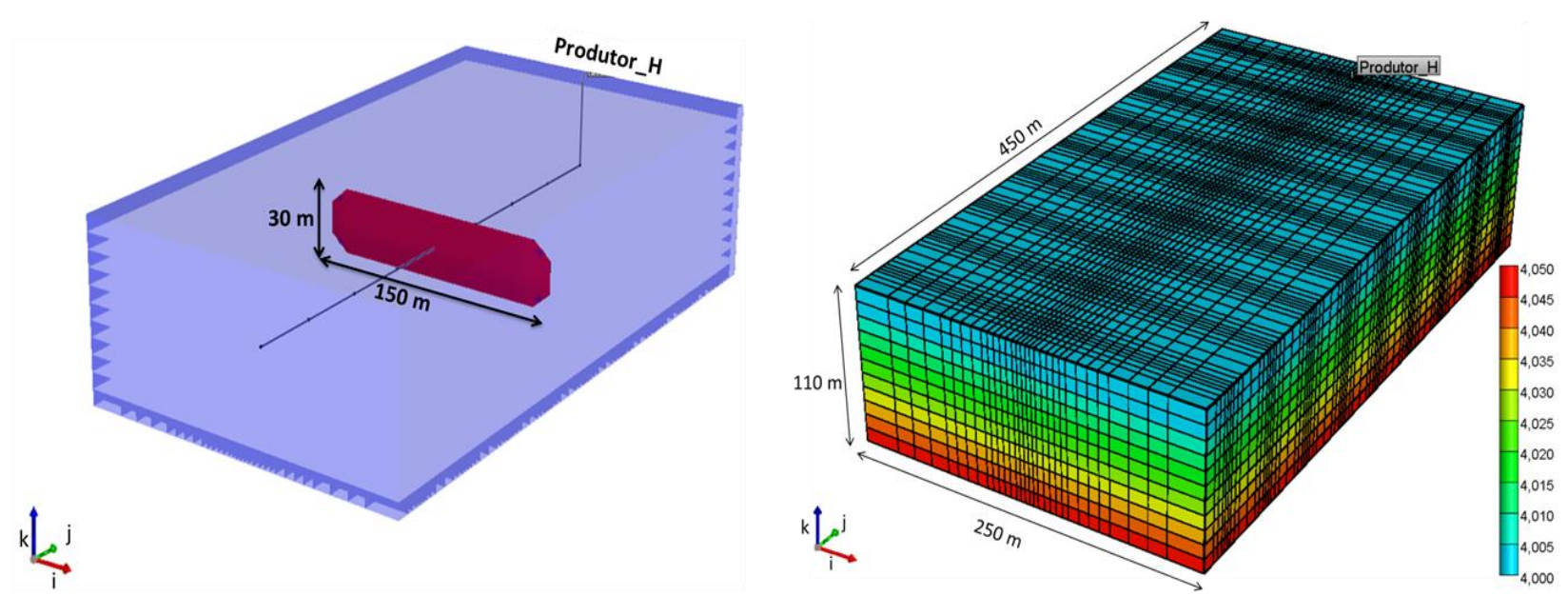

Figura 2 - Modelagem do reservatório e fratura em 3D.

Para simular uma fratura no módulo IMEX, foi preciso refinar as camadas para obter um tamanho ideal, pois normalmente as fraturas possuem cerca de $3 \mathrm{~mm}$ de espessura, porém para atingir um refinamento onde o bloco alcance $3 \mathrm{~mm}$, se faz necessário a utilização de muitos blocos e consequentemente maior tempo de simulação. Outro problema encontrado com o tamanho dos blocos é o diâmetro do poço, tendo em vista de que o tamanho dos blocos não possa ser menor que o diâmetro externo do poço.

Para viabilizar o processo, utilizou-se a equação da condutividade da fratura. Onde a condutividade da fratura é o produto da espessura média da fratura vezes a permeabilidade do propante (SANTOS, 2010), sendo dada pela Equação 1:

$$
C_{f}=\bar{w}_{f}, K_{p}
$$

Onde:

$C_{f}$ : Condutividade da fratura propada convencional (mD.mm)

$\bar{w}_{f}$ : Espessura média da fratura $(\mathrm{mm})$

$K_{\mathrm{p}}:$ Permeabilidade do propante $(\mathrm{mD})$

O modelo original da fratura era com uma espessura de $1 \mathrm{~cm}(10 \mathrm{~mm}) \mathrm{com}$ permeabilidade do propante de $8.000 \mathrm{mD}$, para viabilizar o modelo optou-se por uma espessura de $60 \mathrm{~cm}(600 \mathrm{~mm})$ com uma permeabilidade de $133,4 \mathrm{mD}$, mantendo-se a mesma condutividade.

Neste estudo, a modelagem de fluidos foi realizada utilizando um modelo semissintético, ajustado em 10 pseudo-componentes. A Tabela 2 apresenta as composições dos componentes do fluido. 
Tabela 2 - Agrupamento e fração mássica inicial

\begin{tabular}{cc}
\hline Pseudo-Componentes & Fração Molar \\
\hline CO2 & 0,0001 \\
N2 & 0,001 \\
C1 & 0,689 \\
C2 & 0,086 \\
C3 & 0,053 \\
IC4 & 0,011 \\
NC4 & 0,023 \\
IC5 & 0,009 \\
NC5 & 0,008 \\
FC6 & 0,017 \\
C07 & 0,019 \\
C08 & 0,015 \\
C09 & 0,012 \\
C10+ & 0,052 \\
Total & 1 \\
\hline
\end{tabular}

Os intervalos dos parâmetros analisados são apresentados na Tabela 3.

Tabela 3 - Variáveis operacionais para analise de sensibilidade

\begin{tabular}{cccc}
\hline Parâmetro & $\begin{array}{c}\text { Mínimo } \\
(-1)\end{array}$ & $\begin{array}{c}\text { Intermediário } \\
(0)\end{array}$ & $\begin{array}{c}\text { Máximo } \\
(+1)\end{array}$ \\
\hline Comprimento das fraturas, CF (m) & 50 & 150 & 250 \\
Quantidade de fraturas, QF (und) & 1 & 5 & 9 \\
Permeabilidade da matriz, Kz (mD) & 0,0005 & 0,001 & 0,002 \\
\hline
\end{tabular}

Na Tabela 3, são apresentados os parâmetros operacionais que foram escolhidos para estudar a sensibilidade do processo de fraturamento hidráulico em reservatório com baixas permeabilidades. Este estudo foi realizado através de um planejamento fatorial completo de quatro variáveis com três níveis (33), resultando num total de vinte e sete simulações em função do percentual de gás recuperado em 40 anos. 


\section{Resultados e discussões}

Na Figura 3 é apresentado o diagrama de Pareto para o fator de recuperação do gás (FR), em 40 anos de produção.

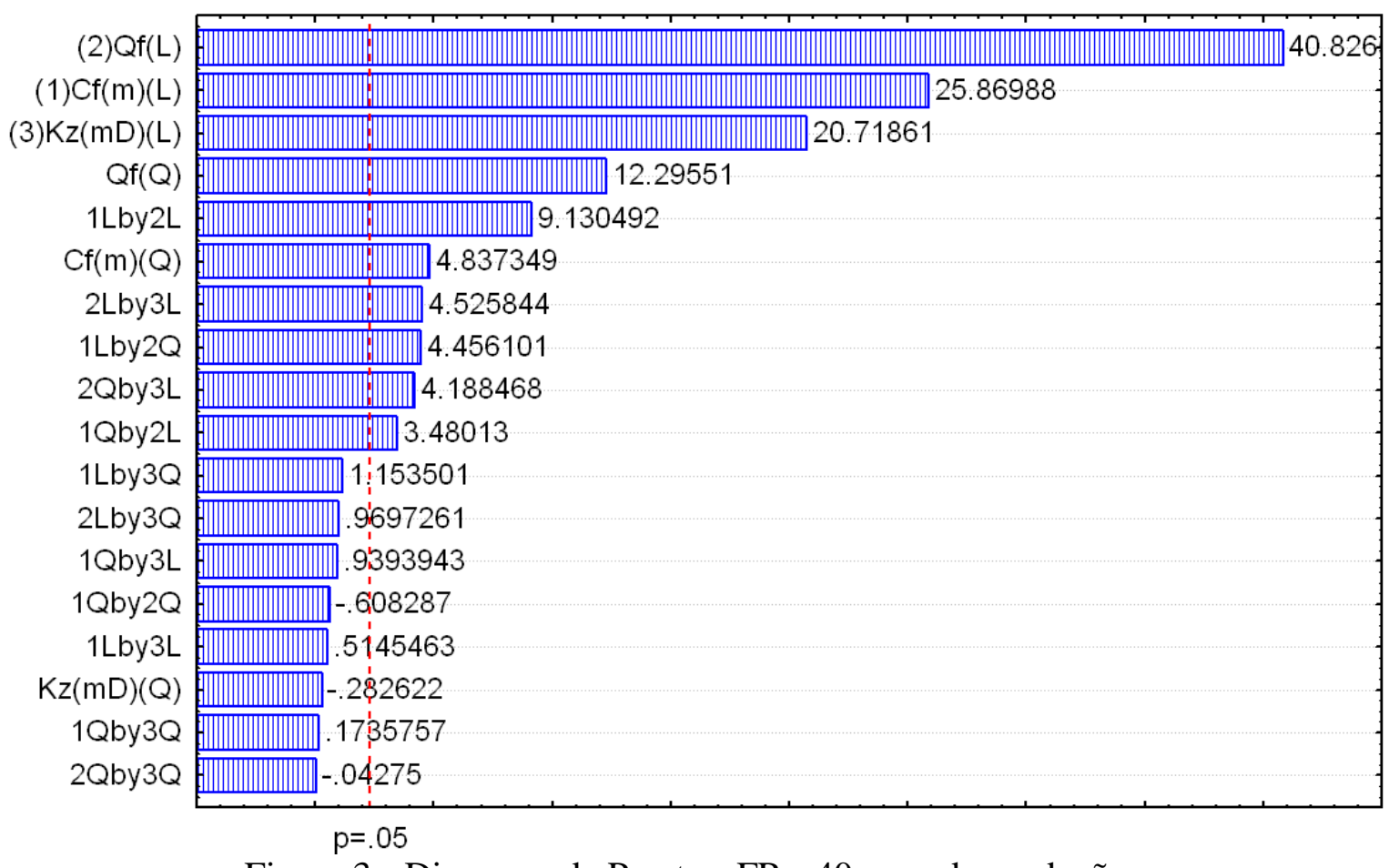

Figura 3 - Diagrama de Pareto - FR - 40 anos de produção.

Observou-se que todos os parâmetros analisados individualmente no processo foram significativos e influenciaram positivamente no fator de recuperação. Analisando-se cada um dos parâmetros operacionais em relação à influência do fator de recuperação, observa-se que:

- O número de fraturas foi o parâmetro que mais contribuiu para o aumento do fator de recuperação para o modelo estudado. Esta resposta mostra que quanto maior a quantidade de fraturas no reservatório, melhor o escoamento do gás no meio poroso para o poço.

- O comprimento das fraturas foi o segundo parâmetros que mais influenciou, para o aumento do fator de recuperação. Demonstrando que quanto maior a fratura, maior a área aberta ao fluxo no reservatório, consequentemente melhorando a produção do gás. 
- A permeabilidade da matriz foi o terceiro parâmetro que mais influenciou na variável resposta.

Na Figura 4, mostra a superfície de resposta com o objetivo de mostrar as influências das interações entre os parâmetros analisados, para identificar a máxima e mínima resposta sobre o fator de recuperação.
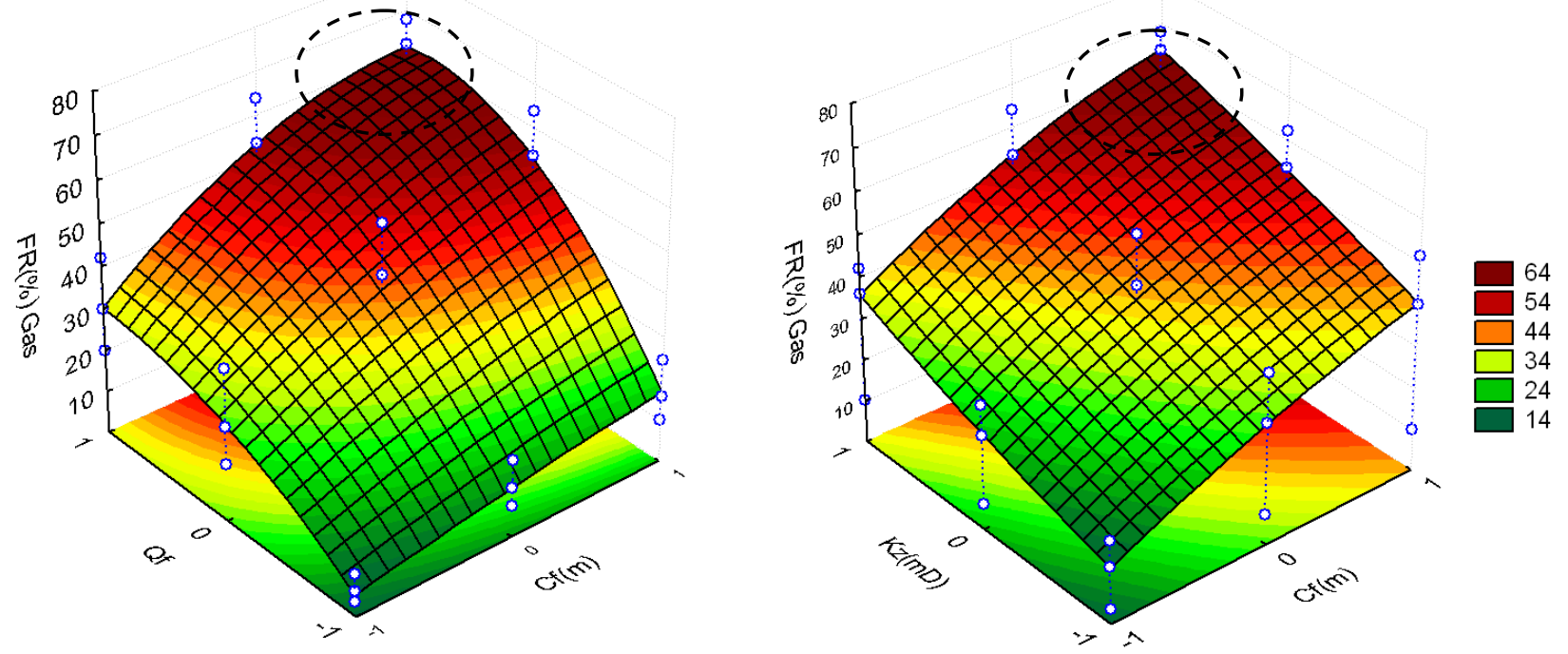

Figura 4 - Superfícies de resposta do FR após 40 anos de produção - Interações entre:

comprimento da fratura versus quantidade de fratura e comprimento da fratura versus permeabilidade da matriz.

A Figura 4 (lado esquerdo) apresenta a superfície de resposta da interação entre o comprimento da fratura com a quantidade de fratura (1L_2L), para o percentual de gás recuperado em 40 anos. Pode ser observado que a região considerada como ótima (região vermelha que apresenta maior percentual de gás recuperado) foi obtida para maiores números de fraturas e para maiores comprimentos de fraturas. Na Figura 4 (lado direito) observa-se que a melhor resposta foi obtida através da interação comprimento da fratura (nível +1) com permeabilidade da fratura (nível +1) indicado pelo circulo preto, observado em ambas as interações. 


\section{CONCLUSÕES}

As principais conclusões obtidas deste estudo foram:

- O estudo realizado comprovou a importante ação promovida pelo fraturamento hidráulico em reservatórios de baixa permeabilidade sobre a recuperação de gás, variando aproximadamente em 67 pontos percentuais no FR em relação à recuperação primária;

- Todos os parâmetros analisados individualmente obtiveram resultados positivamente, aumentando o fator de recuperação do gás;

- Na análise do diagrama de Pareto, o parâmetro que mostrou maior influencia estatisticamente significativo ao um nível de confiança de $95 \%$, foi a Quantidade de Fratura (Qf).

\section{AGRADECIMENTOS}

Ao programa PRH -PB221 pelo suporte financeiro, ao LEAP (laboratório de estudos avançados de petróleo), à CMG (Computer Modelling Group) pelo fornecimento dos programas usados.

\section{REFERENCIAS}

CASTRO, ANTÔNIO ORESTES SALVO: "Seleção de Poços de Petróleo para Operação de Fraturamento Hidráulico: Uma Abordagem Comparativa entre Sistemas Fuzzy-genético e NeuroFuzzy”, Rio de Janeiro, UFRJ (2005).

CHARLES, J, G.; HYDOCK, K, D.; THOMAS, H. Stimulation Evaluation in the Appalachian Tight Gas Sands. SPE 11257, Washington, Novembro, 1982.

ECONOMIDES, J. M. Unified Fracture Desig, Estados Unidos, Orsa Press, 2000.

NEHRING, R. - Growing and Indispensable: The Constribution of Production of Tight Gas Sands to U.S. Gas Production. In: S.P. Cumella, K. W. Shanley, and W. K. Camp, Eds, Understanding, exploring and developing tight-gas sands. Vail Hedber Conference: AAPG Hedber Series, no 3, p 5-12, 2008. 